\title{
INFLUENCIA DEL CAMBIO CLIMÁTICO EN EL TURISMO DE NIEVE DEL PIRINEO. EXPERIENCIA DEL PROYECTO DE INVESTIGACIÓN NIVOPYR
}

\section{Climate change influence on winter tourism in the Pyrenees. Experience from the NIVOPYR research project}

\author{
M. Pons ${ }^{1,2 *}$ J. I. López Moreno³, P. Esteban4, S. Macià ${ }^{4}$ J. Gavaldà5, C. García ${ }^{6}$, M. Rosas-Casals² \& E. Jover ${ }^{1}$ \\ 'Observatori de la Sostenibilitat d'Andorra (OBSA) \\ ${ }^{2}$ Sustainability Measurement and Modeling Laboratory de la Universitat Politècnica de Catalunya (SUMMLAB-UPC) \\ ${ }^{3}$ Instituto Pirenaico de Ecología del Consejo Superior de Investigaciones Científicas (IPE-CSIC) \\ ${ }^{4}$ Centre d'Estudis de la Neu i la Muntanya d'Andorra del Institut d'Estudis Andorrans (CENMA-IEA) \\ ${ }^{5}$ Centre de Lauegi d'Aran, Conselh Generau d'Aran \\ ${ }^{6}$ Institut Cartogràfic i Geològic de Catalunya (ICGC) \\ *Autor de contacto: mpons@obsa.ad
}

Recibido: 07-03-2014. Aceptado: 26-05-2014. Fecha de publicación on-line: 21-10-2014

Citation / Cómo citar este artículo: Pons, M., López-Moreno, J. I., Esteban, P., Macià, S., Gavaldà, J., García, C., Rosas, M. \& Jover, E. (2014). Influencia del cambio climático en el turismo de nieve del Pirineo. Experiencia del proyecto de investigación NIVOPYR. Pirineos, 169, e006. doi: http://dx.doi.org/10.3989/Pirineos.2014.169006

RESUMEN: En los últimos años multitud de estudios han evidenciado y corroborado la existencia de un cambio climático global. A pesar de la existencia de una gran heterogeneidad regional en el grado y la temporalidad de los posibles impactos del cambio climático, cada vez se conocen mejor cuales pueden ser las tendencias futuras sobre posibles cambios en regímenes de temperatura y precipitaciones y, por lo tanto, en otros impactos indirectos sobre la Biosfera o la Criosfera. En este contexto, las áreas de montaña han sido identificadas como regiones especialmente vulnerables a los efectos del cambio climático y como zonas de gran interés para la detección y evaluación de los posibles impactos. Por otro lado, en las últimas décadas, el turismo de invierno, altamente sensible a los cambios en la meteorología y la disponibilidad de nieve, se ha convertido en una de las principales actividades económicas en muchas zonas de montaña y ha jugado un papel clave y fundamental como fuente de ingresos y desarrollo local. Un claro ejemplo es el caso de los Pirineos, una de las regiones europeas más importantes en lo que se refiere a turismo de invierno después de los Alpes.

La motivación principal del proyecto NIVOPYR, proyecto de investigación multinacional en el marco de la Comunidad de Trabajo de los Pirineos (CTP), es evaluar la posible influencia del cambio climático en la evolución del turismo de nieve, y más específicamente el esquí alpino, en esta región. Así, se han realizado diferentes tareas orientadas a integrar el conocimiento ya existente en los patrones presentes y futuros de las temperaturas, precipitaciones, cubierta de nieve y comportamiento de los esquiadores, desarrollar metodologías de análisis y obtener por primera vez datos objetivos para la región Pirenaica. De este modo, se ha obtenido tres perfiles diferentes de estaciones de esquí dependiendo de su vulnerabilidad frente al cambio climático. Un primer grupo de estaciones, identificado como altamente vulnerables, se vería afectado tanto por un escenario de cambio climático medio como por uno más intensivo y, por lo tanto, deberían contemplar la aplicación de medidas de adaptación estructurales, como una mayor desestacionalización y diversificación de su actividad turística. Las estaciones del segundo grupo, consideradas de 
baja vulnerabilidad, podrían seguir operando con medidas de adaptación técnicas bajo un escenario de cambio climático medio, pero no bajo un escenario de cambio climático más intenso. Finalmente, el tercer grupo, engloba las estaciones consideradas como resilientes. Estas estaciones, debido a una situación geográfica y socioeconómica privilegiada frente al resto de estaciones del Pirineo, se verían poco afectadas tanto por un escenario medio de cambio climático como por uno más intenso.

PALABRAS CLAVE: Cambio climático; cobertura de nieve; turismo de invierno; Pirineos; adaptación; variabilidad climática.

\begin{abstract}
In the last decades, several studies have demonstrated and given a valuable insight about the existence of a global climate change. Even though the existence of a high regional heterogeneity about the level and temporality of climate change impacts, the trends of the potential future changes on the temperature and precipitation patterns are better known and consequently the potential impacts on the Biosphere and the Cryosphere. In this context, mountain regions have been identified as highly vulnerable areas to the effects of climate change and especially interesting areas for the detection and assessment of the potential changes and impacts. Moreover, in the last few decades, winter tourism, highly dependent on weather and snow availability, has become one of the main economic activities and source of local development in many mountain regions around the world. The Pyrenees, one of the most important winter tourism areas in Europe after the Alps, is a clear example of this pattern.

The aim of the NIVOPYR project, an international research project in the framework of the Working Community of the Pyrenees (CTP), is to analyze the effects of the climate change on the winter tourism, and especially alpine ski tourism, in the Pyrenees. In order to achieve this objective the project intended to joint the current knowledge about the effects of climate change on temperatures, precipitations, snow cover and skiers behavior in the Pyrenees and develop for first time objective and accurate results for this area. To achieve this goal several methods have been used including, historical analysis of climatical series evolution, assessment of different future climate change scenarios for the Pyrenees, modeling future snowpack based on surface energy balance models and agent based modeling for coupling physical and socioeconomic parameters. One of the main results of this project was the identification of different ski resorts profiles depending on their vulnerability to climate change. Three different groups were identified. The first group includes the high vulnerable ski resorts, effected both by a mid and a high-climate change scenario. The second group, includes the low vulnerable ski resorts, affected by a high-climate change scenario but able to be viable with technical adaptation measures in a mid-climate change scenario. Finally, the group of resilient ski resorts includes the geographically and socioeconomically privileged compared to the rest of Pyrenean ski resorts. These ski resorts would be viable both in a mid and a high-climate change scenario just applying technical adaptation strategies.
\end{abstract}

KEYWORDS: Climate Change; snow cover; Winter tourism; Pyrenees; Adaptation; Climate uncertainty.

\section{Introducción}

En las últimas décadas, el turismo de invierno y de nieve se ha convertido en una de las principales actividades económicas en muchas zonas de montaña y ha jugado un papel clave y fundamental como fuente de ingreso y desarrollo local (WTO-UNEP, 2003; Lasanta et al., 2007). Se estima que el turismo de invierno, principalmente el generado por las estaciones de esquí alpino, mueve anualmente unos 400 millones de visitantes en las principales cordilleras del mundo (Vanat, 2013). Un claro ejemplo es el caso de los Pirineos, una de las regiones europeas más importantes en lo que se refiere a turismo de invierno después de los Alpes. Con unos 11 millones de visitantes al año repartidos en las más de 50 estaciones de esquí de fondo y alpino españolas, francesas y ando- rranas, el sector del turismo de nieve, ya sea de forma directa o indirecta, actúa como principal actividad económica y de desarrollo para gran parte de las comarcas y regiones pirenaicas que en gran medida se sustentan y dependen de este sector ${ }^{1}$. En el caso del Pirineo, el impacto socioeconómico de las estaciones de esquí ha sido desigual, a la vez que ha conllevado unos impactos ambientales asociados al abandono del sector primario y una transición hacia una economía fuertemente dependiente del turismo de esquí principalmente en las proximidades de las estaciones (Lasanta et al., 2007b).

Por otro lado, en los últimos años multitud de estudios han evidenciado y corroborado la existencia de un cambio climático global (IPCC, 2013). A pesar de la existencia de una gran heterogeneidad regional en el grado y la temporalidad de los posibles impactos del cambio climá-

${ }^{1}$ Datos propios obtenidos a partir de hemeroteca, DSF, 2012, ATUDEM, 2013 y SkiAndorra 2013. 
tico, cada vez se conocen mejor cuáles pueden ser los posibles cambios en regímenes de temperatura y precipitaciones y por lo tanto en otros impactos indirectos en la Biosfera o la Criosfera. En este contexto, las zonas de montaña han sido identificadas como regiones especialmente vulnerables a los efectos del cambio climático (Beniston, 2003). Por un lado, debido al ritmo al que se están produciendo estos cambios, por ejemplo en el incremento de la temperatura media terrestre, superior a la media global (El Kenawy, 2012; Esteban et al., 2012). Por otro lado, el gradiente altitudinal propio de los territorios de montaña induce a una gran variedad fisiológica y biológica con abruptos cambios en la fauna y la vegetación y con una gran sensibilidad a pequeñas variaciones climáticas (Keller, 2000). Finalmente, el fuerte vínculo existente entre sistemas naturales y sociales en las zonas de montaña conlleva que el impacto de los cambios ambientales sea mucho más evidente y directo que en otras zonas. Cambios en los regímenes de precipitaciones y, por lo tanto, en los patrones hidrológicos pueden alterar la capacidad de producción hidroeléctrica, o cambios en la ocurrencia de fenómenos extremos pueden inducir un incremento de los impactos socioeconómicos derivados de los riesgos naturales como los aludes o los deslizamientos. Sin embargo, uno de los ejemplos más evidentes es la vulnerabilidad del turismo de nieve frente a los posibles efectos del cambio climático. A pesar de que el cambio climático es sólo uno de los factores que influyen en el turismo de invierno, la presencia o ausencia de nieve, en concreto el espesor de nieve y su temporalidad son elementos claves a la hora de valorar la viabilidad y la sostenibilidad a medio y largo plazo del sector. Por este motivo, la evaluación de los efectos del cambio climático sobre la cobertura natural y la capacidad de producción de nieve es vital y necesaria para valorar los posibles impactos socioeconómicos en una región tan dependiente de esta actividad como los Pirineos.

Hasta la fecha, diversos estudios han analizado con mayor o menor detalle la afectación del cambio climático en algunas de las principales regiones alpinas del mundo. La mayoría de estos estudios se han centrado en los Alpes (König \& Abegg, 1997; Breiling \& Charamza, 1999; Elsasser \& Bürki, 2002; Abegg et al, 2007; Steiger, 2012; Steiger \& Abegg, 2013; Uhlmann et al., 2009), Canadá (McBoyle \& Wall, 1987; Scott et al., 2003; 2007) y los Estados Unidos (Dawson \& Scott, 2010; Dawson et al., 2013), aunque países como Suecia (Moen \& Fredman, 2007), Australia (Hennessy et al., 2003; Bicknell \& McManus, 2006), Japón (Fukushima et al., 2003) o Nueva Zelanda (Hendrikx et al., 2012) también han sido analizados. Sin embargo, en el momento de iniciar este proyecto, no existía ningún estudio científico que analizara la influencia del cambio climático en el turismo de invierno en los Pirineos.

Algunos de estos estudios, principalmente enfocados a analizar los impactos en la oferta (estaciones de esquí), solo tienen en cuenta en sus modelos la nieve natural (Uhlmann et al., 2009) o utilizan indicadores que no son relevantes para las estaciones de esquí como por ejemplo los días de cubierta de nieve, definidos con un umbral de $2,5 \mathrm{~cm}$ de nieve (Lamothe \& Périard, 1988), cuando las estaciones de esquí requieren espesores de entre 20-100 $\mathrm{cm}$ de nieve para que una pista de esquí sea practicable (Scott et al., 2012). Con la excepción de algún estudio, que utiliza modelos estadísticos relacionando el espesor de nieve con otros parámetros climatológicos, (Moen \& Fredmand, 2007), la mayoría utilizan modelos físicos de nieve. Una de las mayores limitaciones del uso de modelos estadísticos es que omiten el efecto de los sistemas de producción de nieve en la disponibilidad de nieve. Esta es la principal limitación, no solo presente en los estudios con modelos estadísticos sino también en algunos que emplean modelos físicos, de la mayoría de estudios que analizan la vulnerabilidad de las estaciones de esquí (Scott et al., 2012). Este aspecto, es clave ya que los estudios que omiten los efectos, tanto positivos como negativos de la producción de nieve artificial no permiten reflejar la realidad de la situación en la mayoría de estaciones de esquí de todo el mundo. En la actualidad, gran parte de los dominios esquiables están equipados con sistemas de producción de nieve y el porcentaje de pistas cubiertas se ve incrementado año a año (Steiger \& Mayer, 2008). Los estudios que incorporan este aspecto (Scott et al., 2003, 2007; Hennessy et al., 2003; Steiger, 2010) muestran en diferentes regiones que el impacto previsto en estudios previos, teniendo en cuenta solo la nieve natural, sería en realidad menor cuando se tiene en consideración la posibilidad de producir nieve. A pesar de estas limitaciones y la heterogeneidad de metodologías utilizadas, la mayoría de estos estudios son congruentes en señalar que un futuro cambio climático podría conllevar un impacto negativo en la duración de la temporada de esquí, la reducción de zonas esquiables y una disminución del número de esquiadores tanto en estaciones de baja altitud como de baja latitud.

En este contexto, la motivación principal del proyecto NIVOPYR, proyecto de investigación multinacional en el marco de la Comunidad de Trabajo de los Pirineos (CTP), es la de evaluar la posible influencia del cambio climático en la evolución del turismo de nieve en esta región. El proyecto se ha centrado en el esquí alpino debido a que es el que genera un mayor impacto socioeconómico. Así se han realizado diferentes tareas orientadas a integrar el conocimiento ya existente, desarrollar metodologías de análisis y obtener nuevos datos necesarios para responder a este objetivo.

La primera tarea era poner en común el conocimiento y el trabajo que en los últimos años diferentes instituciones de investigación han desarrollado sobre el estudio del clima en los Pirineos y, en especial, en lo referente a la variabilidad y disponibilidad de la nieve y sus repercusiones ambientales y socioeconómicas. Por un lado, el proyecto ha permitido recopilar e integrar los registros históricos de las estaciones disponibles en los Pirineos. Por otro lado, se han calculado indicadores climáticos y nivológicos para diferentes regiones del Pirineo y se han aplicado metodologías como el análisis de imágenes de satélite, para ayudar a la comprensión, gra- 
cias a su evolución temporal, tanto de la variabilidad climática natural como de la influencia del cambio climático, haciendo especial énfasis en la evolución del manto nivoso. Este conjunto de datos permite una mejor comprensión de las diferencias en los cambios que se están produciendo en los Pirineos respecto a otras regiones de montaña así como la variabilidad dentro de la misma cordillera pirenaica.

En una segunda fase, se han recopilado todos los datos referentes a escenarios de cambio climático a partir de los modelos regionales disponibles para los Pirineos. A partir de sus proyecciones se ha realizado una detallada clasificación comparativa de variables físicas como las temperaturas, precipitaciones o espesores de nieve en función de los diferentes escenarios de emisiones y el horizonte temporal de las proyecciones. Con el fin de entender mejor la variabilidad futura del manto nivoso en los Pirineos, en esta segunda fase del proyecto se ha realizado una síntesis general de cómo el cambio climático puede afectar concretamente al manto de nieve del Pirineo. Además, se han realizado simulaciones del balance de energía bajo condiciones observadas y distintos escenarios de cambio climático mediante análisis de sensibilidad o utilizando proyecciones de modelos climáticos regionales (RCM's).

Finalmente, se han vinculado los cambios físicos sobre el manto de nieve con la vulnerabilidad futura de las estaciones de esquí. Una de las grandes carencias en la comprensión de los posibles impactos del cambio climático a nivel socioeconómico ha sido la falta de herramientas que permitan proyectar de forma objetiva las posibles repercusiones tanto sociales como económicas de los impactos físicos del cambio climático. De este modo, el objetivo final del proyecto ha sido comprender mejor y vincular de forma objetiva los posibles cambios sobre el manto de nieve y las capacidades futuras de producción de nieve con la vulnerabilidad resultante de las estaciones de esquí de los Pirineos. Esta fase ha permitido crear una herramienta de ayuda a la planificación y toma de decisiones en dominios esquiables tanto para las administraciones públicas como para las empresas privadas del sector.

\section{Estructura del proyecto}

Este trabajo es el resultado de un proyecto de investigación de la CTP y más específicamente de su convocatoria del 2010 (desarrollo del proyecto durante los años 2011 y 2012). Las regiones y países incluidos en este proyecto fueron Andorra, España (Aragón y Cataluña) y Francia. Más concretamente el ámbito geográfico del proyecto incluye las 49 estaciones de esquí alpino más importantes del Pirineo concentradas en una franja central limitada al oeste por La Pierre Saint-Martin y al este por Vallter 2000.

Una de las peculiaridades de este proyecto es que integró tipologías muy variadas de actores. De hecho se organizaron los participantes en tres grupos de trabajo:
- El grupo físico aglutinaba a los climatólogos y debía aportar datos relacionados tanto con la variabilidad histórica de parámetros climáticos en los Pirineos como la posible influencia del cambio climático en estas variables.

- El grupo técnico, formado por profesionales de la nieve, tenía como objetivo facilitar al resto de participantes los datos socioeconómicos relacionados con el turismo de nieve, tales como la frecuentación de esquiadores o los días de apertura, así como identificar las variables técnicas que condicionan la viabilidad de las estaciones de esquí, por ejemplo el mínimo de espesor requerido para que una pista de esquí sea operativa.

- El grupo de modelización tenía por objetivo correlacionar los datos provenientes tanto del grupo físico como del grupo técnico. Para este fin se han utilizado diferentes metodologías como la estadística inferencial, la dinámica de sistemas y la modelización conjunta de modelos por agentes integrados en Sistemas de Información Geográfica.

La estructura de este proyecto fue concebida con el objetivo de maximizar el realismo y la aplicabilidad de los modelos desarrollados. En la Figura 1 se puede observar el mapa conceptual del trabajo dónde se identifican dos grupos de variables. La primera parte del modelo tiene por objetivo evaluar el espesor de nieve a diferentes cotas altitudinales y diferentes zonas de los Pirineos para identificar las zonas que permiten garantizar los $30 \mathrm{~cm}$ de nieve, que es uno de los criterios internacionalmente reconocidos de viabilidad de una estación de esquí (Witmer, 1986). En este sentido, no sólo se ha considerado la nieve natural sino también la potencialidad de producción de nieve de cultivo. La segunda parte del modelo incluye tanto las variables socioeconómicas relacionadas con la actividad del esquí como la respuesta adaptativa de los esquiadores al cambio climático.

\section{Resultados}

\subsection{Ejemplo de reconstrucción histórica}

En cualquier estudio de cambio climático es imprescindible tener un buen conocimiento de la evolución histórica del clima. Esto puede ser una dificultad si hacemos referencia a zonas de alta montaña del Pirineo, dónde escasean las series de datos y en mayor medida los datos de calidad. En el marco de este proyecto, se ha aprovechado para revisar y corregir varias series de alta montaña del Pirineo Catalán. También se ha creado una serie única a partir de los datos de La Molina y de Vall de Núria con la finalidad de tener una serie continua entre los años 1955 y 2012. Otra forma de estudiar la evolución del clima es a través de indicadores climáticos de referencia. Este tipo de datos ayudan a identificar las evidencias del cambio climático. Algunos de los resultados obtenidos en el marco de este proyecto ya han sido publicados en esta misma revista (Esteban et al., 2012). 


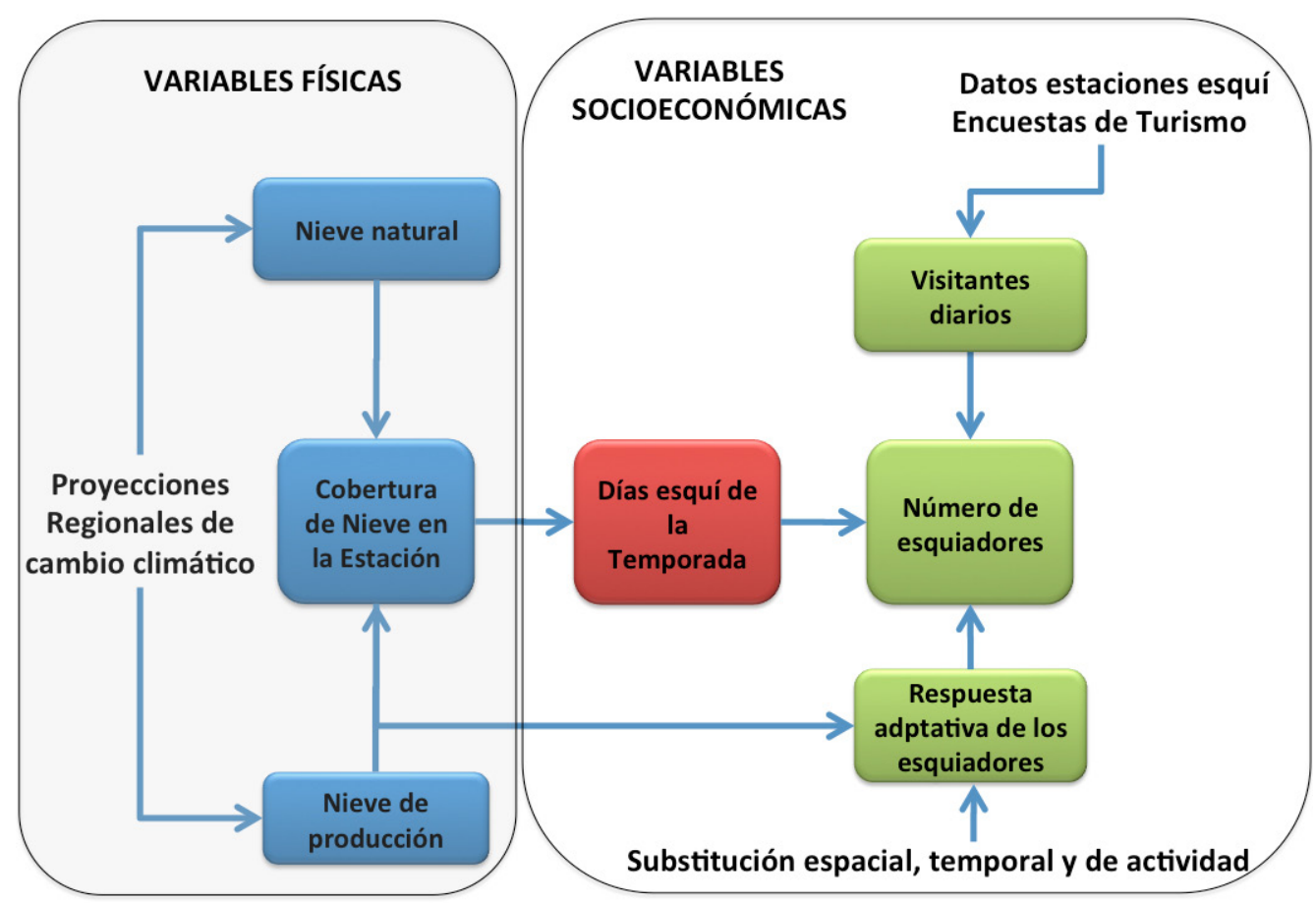

Figura 1. Diagrama del proyecto y del modelo desarrollado.

Figure 1. Diagrams of the project and developed model

\subsection{Recopilación de escenarios existentes}

El conocimiento de las tendencias históricas de la evolución climática del Pirineo genera datos muy relevantes. Sin embargo, en el marco de este proyecto era necesario evaluar también sus posibles evoluciones futuras. Cada modelo climático se basa en unas hipótesis y metodologías de trabajo que lo hacen único. En este sentido, es interesante usar diversos modelos sumando así sus diferentes perspectivas y abordar de la manera más sólida posible el reto de la incertidumbre. Con este objetivo se han analizado los diferentes modelos de cambio climático existentes para el Pirineo, considerando tanto modelos dinámicos como proyecciones estadísticas derivadas. En este sentido se han usado los modelos siguientes:

- Información facilitada por MeteoFrance, proyecto ANR/SCAMPEI. A partir de las modelizaciones a escala global ARPEGE y LMDZ (resoluciones entre 50 y $300 \mathrm{~km}$ ) se ha pasado a modelos mesoescalares como el ALADIN, el MAR y una versión especial del LMDZ. Además se hicieron correcciones estadísticas de las salidas basándose en análogos gracias a los datos de la red SAFRAN. A partir de aquí se obtienen los resultados para diferentes escenarios de cambio climático y para diferentes horizontes temporales y con una resolución de $8 \mathrm{~km}$. En este trabajo se han analizado los resultados para 30 puntos distribuidos por todo el Pirineo francés (SCAMPEI, 2012).

- Información obtenida a partir de la Agencia Estatal de Meteorología (AEMET) - Generación de escenarios regionalizados de cambio climático para España. De la información disponible se ha trabajado con los resultados derivados de dos proyecciones del CMIP3AR4 IPCC (la del modelo canadiense CCCMA CGM3_1 y el modelo alemán MPI_ECHAM5) y tres del proyecto ENSEMBLES (modelo alemán MPEH5, el noruego BCCR_BCM2 y el francés CNR-CM3). De estas proyecciones se obtienen diferentes escenarios de cambio climático para 19 puntos del Pirineo español, en relación a las temperaturas (mínimas y máximas) y 60 puntos para datos de precipitación (Brunet et al., 2009).

- Información obtenida a partir del Servei Meteorològic de Catalunya (SMC) - proyecto ESCAT (Barrera-Escoda \& Cunillera, 2011; ESCAT, 2012). A partir de las salidas de modelos globales (el alemán ECHAM5), se deriva información de alta resolución a partir del acoplamiento de modelos meteorológicos mesoescalares (MM5) con los de circulación general y así derivar la información a gran escala hasta los $15 \mathrm{~km}$ de resolución. En este trabajo se han considerado valores globales para el Pirineo catalán de temperatura, precipitación, viento y humedad relativa para diferentes escenarios de cambio climático. 


\section{6• M. PONS, J. I. LÓPEZ MORENO, P. ESTEBAN, S. MACIÀ, J. GAVALDÀ, C. GARCÍA, M. ROSAS \& E. JOVER}

Sumando estas diferentes fuentes de información se puede obtener una buena perspectiva de las posibles tendencias de las alteraciones provocadas por el cambio climático en la temperatura y las precipitaciones para el conjunto del Pirineo y considerando una importante variabilidad metodológica. Centrándonos en la temporada invernal y, como ejemplo, presentamos en la siguiente figura la evolución de la temperatura máxima para tres horizontes temporales: cercano (2020-2050), mediano (20402070) y lejano (2070-2100) y para diferentes escenarios de cambio climático (A1B, A1B-1, A1B-2, A2 y B1) (Figura 2). La temperatura máxima muestra un importante impacto de las futuras concentraciones de gases de efecto invernadero con incrementos en todos los puntos y escenarios estudiados. Este incremento medio es más marcado cuanto más lejano es el horizonte temporal, pasando de entre $1,1^{\circ}$ y $1,9^{\circ} \mathrm{C}$ para el horizonte cercano a entre $2,1^{\circ}$ y $4^{\circ} \mathrm{C}$ para el horizonte lejano. También cabe destacar que cuanto más lejana es la proyección mayor es la variabilidad de los resultados, tanto de un mismo modelo en diferentes zonas geográficas como entre diferentes modelos. En definitiva, hay un incremento de la incertidumbre y de la disparidad de los resultados. Así por ejemplo, para el horizonte lejano algún modelo (BCCR_BCM3) muestra sólo incrementos moderados e incluso en algunos puntos inferiores a $+1^{\circ} \mathrm{C}$ cuando el modelo LMDZ muestra resultados que pueden superar $\operatorname{los}+5^{\circ} \mathrm{C}$ en algunos casos.

\subsection{Escenarios especificos de evolución del manto de nieve}

Uno de los objetivos del proyecto era poder determinar qué evolución podría tener el manto de nieve en distintas zonas del Pirineo y para diferentes bandas altitudinales. Con este objetivo se realizaron proyecciones que

\section{TEMPERATURA MÁXIMA (NDEFMA)}

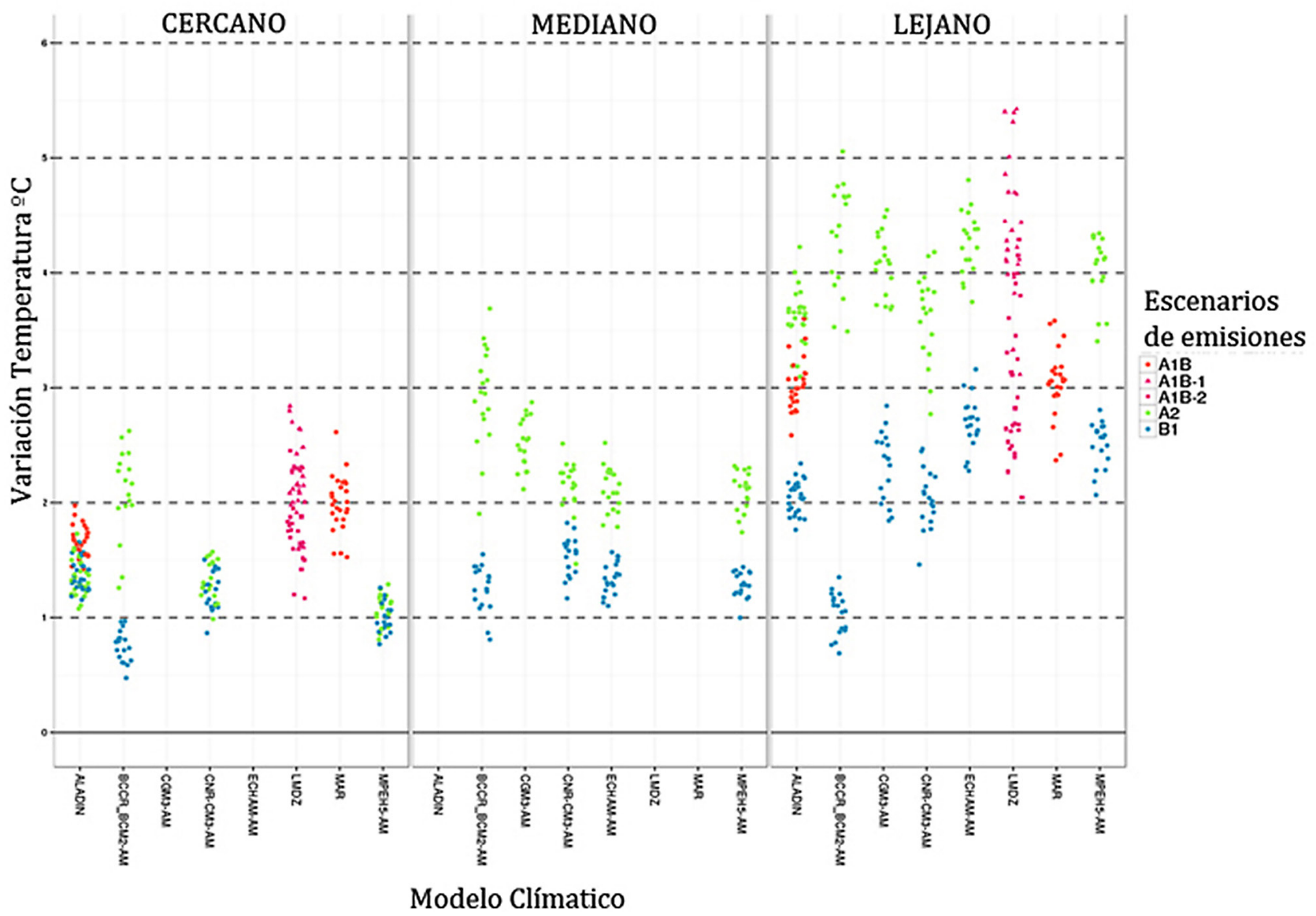

Figura 2. Distribución de la evolución de la temperatura máxima invernal (de noviembre a abril) en varios puntos del Pirineo en función de diferentes modelos, escenarios de emisiones y para varios horizontes temporales. Para abordar el problema de la incertidumbre se han utilizado modelos de distintas fuentes: Météo-France (SCAMPEI) y AEMET.

Figure 2. Distribution of the evolution of maximum winter temperature (November-April) according to different models, emission scenarios and time horizons. Uncertainty has been assesed by using different data sources: Météo-France (SCAMPEI) and AEMET data 
sirvieron como datos de entrada al modelo regional detallado en la siguiente sección. Así por ejemplo se han usado, por un lado, resultados ya publicados sobre proyecciones del manto nivoso en el Pirineo, generados a partir de un modelo de balance de energía de la nieve alimentado por proyecciones futuras, a partir del modelo climático regional HIRHAM (Christensen et al., 1998), de las variables: temperatura del aire, punto de rocío, precipitación, viento, presión en superficie y radiación solar incidente (López-Moreno et al., 2009). Por otro lado, también se han generado datos nuevos obtenidos a partir de la modelización del manto de nieve a $2000 \mathrm{~m}$ de altitud en una estación meteorológica cercana a la estación de esquí de Formigal (Izas a $2.056 \mathrm{~m}$ ). Para ello, se ha utilizado el modelo CRHM (Cold Region Hydrological Model). Los resultados han mostrado cómo bajo los escenarios de cambio climático podemos tener una reducción de entre el 15 y $25 \%$ de acumulación de nieve y entre el 10 y $20 \%$ de su duración (alrededor de un mes). El ritmo de fusión puede verse también notablemente alterado. Con el objetivo de adaptarse a las necesidades de datos físicos como entradas del modelo se han considerado dos escenarios de cambio climático. El primero corresponde a un incremento de $+2^{\circ} \mathrm{C}$, equivalente al escenario B2 del SRES IPCC (IPCC, 2007) y un segundo escenario más extremo con un incremento de $+4^{\circ} \mathrm{C}$, equivalente al escenario $\mathrm{A} 2$. El uso de la nomenclatura $+2^{\circ} \mathrm{C} y+4^{\circ} \mathrm{C}$ para los escenarios se debe principalmente a dos motivos. Por un lado, la referencia solo al cambio de temperatura se debe a que el modelo HIRHAM predice que los cambios más importantes en la cobertura de nieve son causados en mayor medida por temperaturas más elevadas que por cambios en los patrones de precipitaciones. Por otro lado, para evitar hablar de escenarios SRES A2 y B2, con un horizonte temporal 2070-2100, se creyó conveniente simplificarlo con escenarios equivalentes $+2,+4$ grados para simplificar la comunicación hacia las administraciones y gestores de la estaciones de esquí. De este modo, el uso de esta nomenclatura permite equiparar los escenarios futuros a temporadas pasadas con una anomalía climática similar a la proyectada y analizar y concienciar a los actores implicados de los posibles impactos sobre el sector.

\subsection{Modelo agregado de impactos físicos y socioeconómicos del cambio climático}

Una vez analizados cuáles podrían ser los impactos del cambio climático a nivel físico y, en especial, los efectos sobre la cubierta de nieve del Pirineo, el siguiente paso ha sido el desarrollo de un modelo integral a escala regional que permita vincular estos posibles cambios físicos con las repercusiones socioeconómicas que supondrían sobre el turismo de invierno a nivel local y regional (Figura 1). Para este fin se ha creado un modelo georreferenciado basado en agentes (GIS+ABM) acoplado a un modelo gravitacional. El modelo basado en agentes (ABM) permite simular, por un lado, la variación diaria de la cubierta de nieve a nivel local en cada una de las estaciones, y por lo tanto la capacidad de operar o no de dicha estación. De este modo, utilizando las proyecciones de nieve natural de modelos climáticos regionales, se simula diariamente, añadiendo la capacidad de producción de nieve, las condiciones de cada estación para poder operar o no a lo largo de una temporada. Por otro lado, el modelo por agentes permite simular la respuesta adaptativa individual de los esquiadores en función de las condiciones de nieve de las estaciones, como por ejemplo, dejar de esquiar o ir hacia la estación más próxima con nieve disponible. Finalmente, el modelo gravitacional permite en función de la capacidad de atracción de las estaciones de esquí, basada en características tanto físicas (altitud, superficies esquiables,...) como comerciales (precio del forfait, actividades turísticas complementarias,...) y de la distancia entre estaciones, simular la redistribución potencial de esquiadores entre aquellas estaciones que siguen operando. Para analizar los efectos del cambio climático sobre el turismo de nieve del Pirineo, se han aplicado a dicho modelo integral cuatro escenarios diferentes. Un primer escenario asume un incremento de la temperatura media invernal de $2^{\circ} \mathrm{C}$. En un segundo escenario más intensivo de cambio climático, se asume un incremento de $4^{\circ} \mathrm{C}$. Estos dos escenarios solo contemplan la cobertura natural de nieve para determinar la capacidad de operar de las estaciones de esquí y nos permiten evaluar la vulnerabilidad natural de cada una de ellas. Para poder analizar la capacidad de la nieve de producción como medida de adaptación al cambio climático y realizar escenarios de futuro más realistas, ya que a día de hoy gran parte de los dominios esquiables ya disponen de estos sistemas de innivación, se ha incluido el efecto de esta tecnología en los otros dos escenarios. De este modo, se han simulado los efectos bajo un incremento de $2^{\circ} \mathrm{C}$ y $4^{\circ} \mathrm{C}$ teniendo en cuenta la capacidad de producir nieve en estos escenarios climáticos.

\subsubsection{Vulnerabilidad natural y técnica de las estaciones de esquí del Pirineo}

El presente modelo se desarrolló primero a escala local para Andorra (Pons-Pons et al., 2012). El objetivo era probar el buen funcionamiento del modelo en un espacio limitado. Pero estos resultados tienen que considerarse como parciales, ya que existe una importante interacción entre las estaciones (competencia) que sólo se tiene en consideración en un estudio más extenso de ámbito regional. A escala del Pirineo, el primer resultado que se obtiene del modelo integral es el análisis de la vulnerabilidad tanto natural como técnica de las estaciones de esquí de los Pirineos. Para determinar si una estación de esquí es o no viable, se ha utilizado el criterio a día de hoy más extendido en este tipo de análisis: la regla de los $30 \mathrm{~cm}$ durante 100 días (Abegg et al., 2007; Scott et al., 2003; Steiger 2010; Witmer, 1986). Según este criterio, se considera que una estación de esquí es viable cuando dispone de una cubierta de nieve de al menos 30 centímetros durante un mínimo de 100 días por temporada. De este 


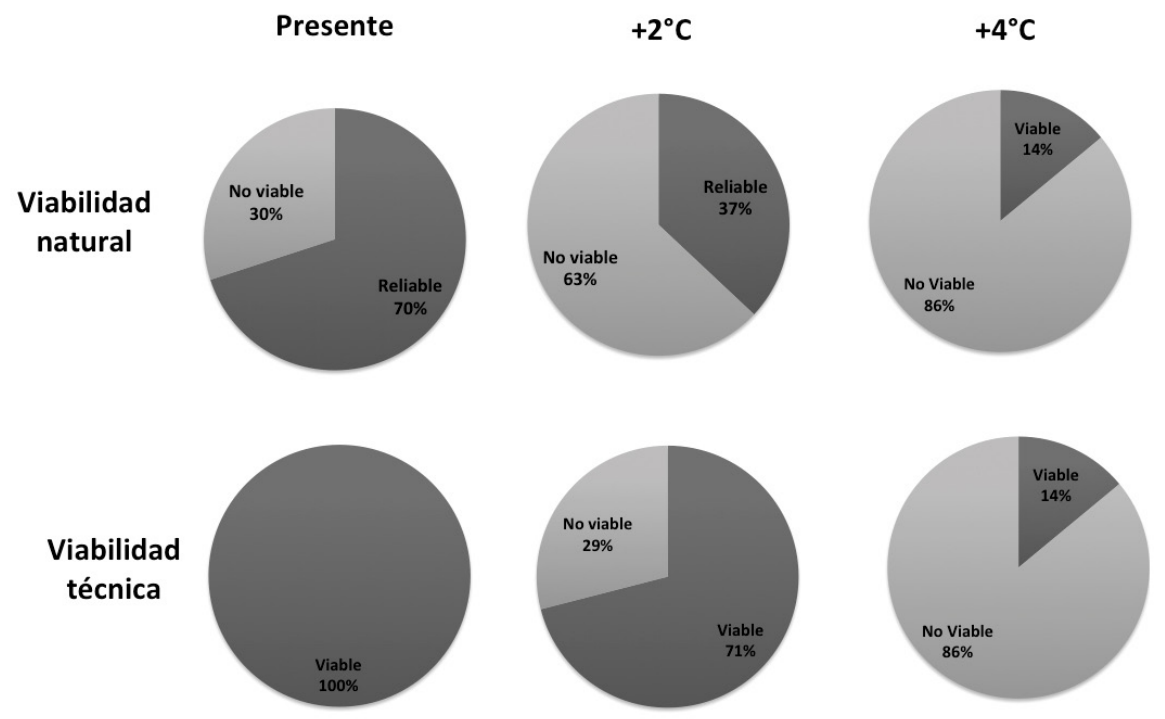

Figura 3. Viabilidad natural y técnica de las estaciones de esquí del Pirineo.

Figure 3: Natural and technique viability of Pyrenean sky resorts

modo, a partir de las proyecciones de cambios en la cubierta de nieve, se ha calculado la cantidad de estaciones viables de forma natural en una temporada promedio presente y bajo dos escenarios de cambio climático $\left(+2^{\circ} \mathrm{C}\right.$ y $+4^{\circ} \mathrm{C}$ ). Para las proyecciones de nieve natural, se han utilizado los resultados de López-Moreno et al., (2009), construidas a partir del modelo GRENBLS (Keller et al., 2005), utilizando como input variables climáticas obtenidas a partir del modelo climático Regional HIRHAM (Christensen et al., 1998). Antes de realizar el análisis se ha efectuado un downscaling espacial de las variables climáticas a cuatro bandas altitudinales: 1500, 2000, 2500 y 3000 metros. Las proyecciones de los cambios en la cubierta de nieve en el Pirineo se han simulado a partir de dos escenarios futuros de emisiones: el SRES A2 y el B2 (IPCC 2007).

Por otro lado, para analizar la viabilidad técnica de las estaciones de esquí, es decir teniendo en cuenta la capacidad de producir nieve, se ha utilizado el mismo criterio pero en este caso considerando el efecto de esta tecnología sobre la cubierta de nieve natural proyectada para cada estación. La Figura 3 muestra la vulnerabilidad natural y técnica (con nieve de producción) de las estaciones de esquí del Pirineo en el presente y bajo dos escenarios de cambio climático, uno medio y uno de más intensidad.

\subsubsection{Impacto sobre la frecuencia de esquiadores}

A partir de la proyección de los días esquiables en cada estación de esquí y de las estadísticas de visitantes obtenidas a partir de datos históricos de las estaciones
(Datos propios obtenidos a partir de hemeroteca; DSF, 2012; ATUDEM, 2013 y SkiAndorra, 2013) y de los datos obtenidos a través de las encuestas de turismo de Andorra (Andorra Turisme, 2012), se ha proyectado la frecuentación de esquiadores en cada estación bajo los diferentes escenarios comentados anteriormente. Hasta día de hoy, la mayoría de estudios internacionales, que han analizado los posibles impactos del cambio climático sobre el turismo de nieve, consideraba que la disminución de esquiadores era proporcional a la disminución de días esquiables. Es decir, cuando una estación se considera no viable, se produce la pérdida del total de esquiadores. Sin embargo, estudios recientes han demostrado que solo una pequeña parte de estos esquiadores, entorno al 4\%, dejaría de esquiar si cerrara la estación de esquí que frecuentan. El resto, mostraría una capacidad adaptativa ya sea mediante una sustitución espacial de la actividad (buscando la estación más cercana con las condiciones deseadas) o temporal (esquiando con más frecuencia durante los periodos que abra la estación) (Behringer et al., 2000; Dawson et al., 2011; Gössling et al., 2012; Dawson et al., 2013). Estos datos muestran la necesidad de incorporar este aspecto desde un punto de vista regional en los análisis de impactos, ya que los impactos del cambio climático sobre la cubierta de nieve pueden ser muy heterogéneos, incluso a distancias muy cortas, lo que hace que la vulnerabilidad de las estaciones de esquí de cualquier región sea muy alta. Para incorporar la capacidad adaptativa de los esquiadores, se ha simulado la sustitución espacial y de actividad de los esquiadores cuando la estación que frecuentaban no dispone de las condiciones mínimas para operar. Como a día de hoy no se dispone de encuestas sobre la respuesta 
adaptativa de los esquiadores en el Pirineo, se han utilizado como referencia los datos de comportamiento de los estudios realizados en los Alpes y Norteamérica (Behringer et al., 2000; Dawson et al., 2011; Gössling et al., 2012; Dawson et al., 2013). Para estimar la sustitución espacial, se ha utilizado un modelo gravitacional georreferenciado que distribuye los esquiadores de una estación de esquí cerrada entre las disponibles en función de dos criterios: la distancia entre las estaciones de esquí y la capacidad de atracción de esquiadores de cada una de ellas. Para modelizar la capacidad de atracción de cada una de las estaciones se ha creado un indicador de atractivo basado en las características físicas de las estaciones como la cota media o los kilómetros esquiables, y características socioeconómicas, como el precio del forfait o la oferta de actividades turísticas complementarias. El indicador creado representa de forma estadísticamente significativa $\left(\mathrm{R}^{2}=0,87 ; \mathrm{p}\right.$-value $\left.<0,01\right)$ la distribución actual de esquiadores entre las estaciones de esquí de los Pirineos. De este modo, cuando una estación de esquí se considera no viable, se estima que un $4 \%$ de sus esquiadores cambia de actividad y el resto se redistribuye entre las estaciones de esquí restantes que seguirían operando siguiendo el modelo gravitacional. En un ejemplo hipotético, el cierre de la estación de la Molina supondría, según el modelo gravitacional, que una gran cantidad de esquiadores se desplazaría hasta Masella, debido al factor de proximidad y viceversa. Sin embargo, a pesar de que GrandValira se encuentra a mayor distancia que otras estaciones abiertas más próximas, se observa que esta estación absorbería un $11 \%$ de los esquiadores de la Molina, debido a su alta capacidad de atracción.

\subsubsection{Clasificación de las estaciones de esquí según el nivel de vulnerabilidad}

El modelo regional descrito nos permite analizar cómo los cambios locales proyectados sobre la cubierta de nieve van a impactar a nivel regional sobre el turismo de nieve en los Pirineos. Dicho análisis nos ha permitido identificar y clasificar en tres grupos las estaciones de esquí del Pirineo según su nivel de vulnerabilidad. El primer grupo está caracterizado por estaciones de esquí con un bajo nivel de atractivo turístico y unas condiciones geográficas y climáticas menos favorables (estaciones a cotas más bajas, con una mayor influencia mediterránea y/o con orientaciones predominantes del Sur,...). Este grupo muestra una disminución en el número de esquiadores tanto en un escenario de cambio climático medio $\left(+2^{\circ} \mathrm{C}\right)$ como severo $\left(+4^{\circ} \mathrm{C}\right)$, incluso con el uso de nieve de producción. Un segundo grupo, con un nivel de atractivo medio y con unas condiciones climáticas y geográficas más favorables que el primer grupo, muestra que bajo un escenario de cambio climático medio es capaz de incrementar la frecuentación de esquiadores en su dominio, en gran medida gracias a la nieve de producción. Sin embargo, bajo un escenario de cambio climático más severo, la mayoría de estaciones sufrirían un descenso de la frecuentación, principalmente debido a la pérdida de días con las condiciones mínimas de temperatura para producir nieve de cultivo y, por lo tanto, de su capacidad para mantener una temporada más larga. Finalmente, el tercer grupo está caracterizado por estaciones con unas características geográficas privilegiadas (mayor altitud, mayor influencia atlántica y/o orientaciones predominantes de Norte) y un mayor factor de atractivo turístico. Este grupo es considerado el más resiliente, ya que a pesar de sufrir una reducción de la temporada de esquí, la capacidad de abrir durante más días que sus competidores y su mayor y más diversa oferta turística, les proporcionan una ventaja estratégica que les permitirá, tanto en un escenario de cambio climático moderado como en uno más severo, atraer a aquellos esquiadores de las estaciones de esquí cercanas más vulnerables.

La figura 4 muestra la evolución de la vulnerabilidad y del volumen de esquiadores bajo diferentes escenarios de cambio climático para tres estaciones del Pirineo de distinta vulnerabilidad al cambio climático.

Estos resultados muestran y cuantifican por primera vez de forma objetiva que la principal consecuencia del cambio climático no será una disminución significativa del turismo de nieve sino una redistribución del turismo de nieve de las estaciones más vulnerables hacia aquellas estaciones más resilientes, teniendo en cuenta no solo la variabilidad física y climática de cada una de las estaciones sino también la influencia de otros factores socioeconómicos como la oferta turística complementaria o la accesibilidad. Por otro lado, esta clasificación permite diferenciar la vulnerabilidad de cada una de ellas y por lo tanto cuáles podrían ser las medidas de adaptación al cambio climático. De este modo, las estaciones de esquí identificadas como resilientes muy probablemente podrán continuar su actividad aplicando medidas de adaptación técnicas como la nieve de producción o la preparación de pistas. Sin embargo, debido a la capacidad de atraer nuevos esquiadores, seguramente deberán aplicar medidas para reducir y controlar el impacto sobre el entorno como consecuencia del incremento de actividad y del uso más intensivo que requerirán las medidas tecnológicas de adaptación, como puede ser el uso más intensivo de los cañones de nieve y sus repercusiones sobre los recursos energéticos e hídricos. El segundo grupo, identificado como de baja vulnerabilidad, podrá basarse a medio plazo en medidas de adaptación técnicas, pero con una planificación futura basada en cambios más estructurales de su oferta turística como una mayor diversificación y desestacionalización de sus actividades. Finalmente, el grupo identificado como altamente vulnerable a los efectos del cambio climático, comprende aquellas estaciones que deberían reconsiderar el turismo de nieve como su actividad principal y reorientarlo, en un marco de turismo sostenible, hacia otras actividades que no dependan tanto del recurso nivoso y estén más orientadas al turismo de montaña estival como por ejemplo el ciclismo de montaña, el turismo rural o el senderismo. 


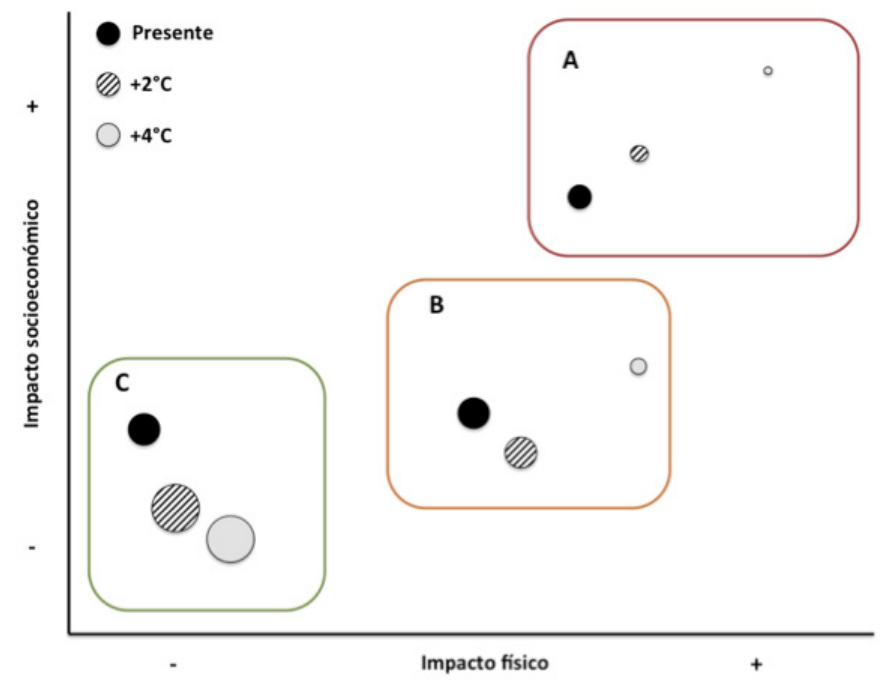

Figura 4. Evolución de los impactos negativos, tanto económicos como físicos, y del volumen de esquiadores (mayor volumen, mayor frecuencia de esquiadores) en las estaciones A (alta vulnerabilidad), B (baja vulnerabilidad) y C (resiliente) bajo diferentes escenarios de cambio climático.

Figure 4. Evolution of econonomic and physical negative impacts and on number of skiers in the ski resorts under different scenarios of climate change. A (high vulnerability), B (low vulnerability) and C (resilient).

\section{Conclusiones}

En este proyecto de la CTP se ha conseguido por primera vez evaluar de forma objetiva la influencia del cambio climático en el turismo de nieve para la región del Pirineo. Debido al importante impacto económico y social de esta actividad era necesario tener un estudio de esta tipología que identificase la vulnerabilidad de las diferentes infraestructuras existentes. Cabe destacar, por un lado, la heterogeneidad y gran variabilidad espacial de los resultados obtenidos, indicando que no se puede generalizar los impactos y posibles soluciones para todas las estaciones. En ese sentido, la buena adaptación al cambio climático del sector pasará por acciones individualizadas para las diferentes estaciones en función del grado de la vulnerabilidad propia de cada una de ellas y del impacto esperado. Para las más resilientes, seguramente será suficiente con medidas de adaptación técnicas para garantizar su actividad. En cambio, las estaciones más vulnerables deberán basarse en soluciones más estructurales, fomentando las actividades complementarias, mejoras en las comunicaciones, la desestacionalización o en los casos más extremos, un completo cambio de actividad. Sin embargo cabe destacar que en la consecución de este proyecto se han identificado una serie de limitaciones y líneas futuras de investigación. Por un lado, la influencia que pueden tener factores como el viento y otros fenómenos meteorológicos extremos en el funcionamiento de las instalaciones y las condiciones de esquiabilidad en una estación. Por otro lado, la importancia de tener en cuenta variables locales como la orografía, la orientación o la pendiente de las pistas, ya que juegan un papel crucial en la distribu- ción espacial de la nieve. En este sentido, en la actualidad se está desarrollando otro proyecto de la CTP: “Creación de un modelo de alta resolución espacial para cuantificar la esquiabilidad y la afluencia turística en el Pirineo bajo diferentes escenarios de cambio climático" con el objetivo de profundizar en estas líneas de trabajo y poder obtener unos resultados con un mayor grado de precisión y definición.

\section{Agradecimientos}

Los autores de este trabajo agradecen la financiación del Govern d'Andorra (CTP-AND/2010), el Gobierno de Aragón y la Generalitat de Catalunya relacionada con el proyecto de la CTP 2010-2012 y el Govern d'Andorra (CTP-AND/2012) y el Gobierno de Aragón la financiación del proyecto CTP2013-2014: "Creación de un modelo de alta resolución espacial para cuantificar la esquiabilidad y la afluencia turística en el Pirineo bajo diferentes escenarios de cambio climático". Marc Pons agradece al Gobierno de Andorra por una beca predoctoral [BTC2012/2013-0006-AND]. Se agradece también la información facilitada por Météo France de los resultados del proyecto SCAMPEI.

\section{Referencias}

Abegg, B., Agrawala, S., Crick, F., \& De Montfalcon, A. 2007. Climate change impacts and adaptation in winter tourism. In S. Agrawala (Ed.): Climate change in the European Alps: 
Adapting winter tourism and natural hazards management (pp. 25-58). Paris: Organization for Economic Cooperation and Development.

Andorra Turisme. 2012. Encuestas sobre actividad turística de los visitantes del Principado de Andorra.

ATUDEM. 2013. Dossier de prensa 2012-2013. Associación Tursítica de Estaciones De Esquí y Montaña.

Barrera-Escoda, A. \& Cunillera, J. 2011. Projeccions de canvi climàtic per a Catalunya (NE Península Ibèrica). Part I: Modelització climàtica regional. Tethys, 8: 77-89, doi: http:// dx.doi.org/10.3369/tethys.2011.8.08.

Behringer, J., Bürki, R. \& Fuhrer, J. 2000. Participatory integrated assessment of adaptation to climate change in alpine tourism and mountain agriculture. Integrated Assessment, 1(3): 331-338

Beniston, M. 2003. Climatic change in mountain regions: A review of possible impacts. Climatic Change, 59: 5-31. doi: http://dx.doi.org/10.1007/978-94-015-1252-7 2

Bicknell., S. \& McManus, P. 2006. The canary in the coalmine: Australian ski resorts and their response to climate change. Geographical Research, 44: 386-400. doi: http://dx.doi. org/10.1111/j.1745-5871.2006.00409.x

Breiling, M. \& Charamza, P. 1999. The impact of global warming on winter tourism and skiing: a regionalized model for Austrian snow conditions. Regional Environmental Change, 1(1):4-14

Brunet, M., Casado, M.J, de Castro, M., Galán, P., López, J. A., Martín, J.M., Pastor, A., Petisco, E., Ramos, P., Ribalaygua, J., Rodríguez, E., Sanz, I. \& Torres, L. 2009. Generación de escenarios regionalizados de cambio climático para España. Ministerio de Medio Ambiente y Medio Rural y Marino; Agencia Estatal de Meteorología: 158 pp., Madrid.

Christensen, O.B., Christensen, J.H., Machenhauer, B. \& Botzet, M., 1998. Very high resolution regional climate simulations over Scandinavia-present climate. Journal of Climate, 11: 3204-3229. doi: http://dx.doi.org/10.1175/1520-0442 (1998)011<3204:VHRRCS >2.0.CO;2

Dawson, J. \& Scott, D. 2010. Systems analysis of climate change vulnerability for the US Northeast ski sector. Tourism Planning and Development, 7(3): 219-235 http://dx.doi.org/ 10.1080/1479053X.2010.502383

Dawson, J., Havitz, M. \& Scott, D. 2011. Behavioral Adaptation of Alpine Skiers to Climate Change: Examining Activity Involvement and Place Loyalty. Journal of Travel \& Tourism Marketing, 28(4): 388-404. http://dx.doi.org/10.1080/10548 408.2011.571573

Dawson, J., Scott, D. \& Havitz, M. 2013. Skier demand and behavioural adaptation to climate change in the US Northeast. Leisure/Loisir, 37(2): 127-143. http://dx.doi.org/10.1080/14 927713.2013.805037

DSF 2012. Recueil d'indicateurs et analyses 2012. Domaines Skiables de France

El Kenawy, A., López-Moreno, J.I. \& Vicente-Serrano, S.M. 2012. Trend and variability of temperature in northeastern Spain (1920-2006): linkage to atmospheric circulation. Atmospheric Research, 106: 159-180. http://dx.doi.org/ 10.1016/j.atmosres.2011.12.006

Elsasser, H. \& Bürki, R. 2002. Climate change as a threat to tourism in the Alps. Climate Research, 20: 253-257. http:// dx.doi.org/10.3354/cr020253

ESCAT, Generació d'escenaris climàtics amb alta resolució per a Catalunya (Resum executiu) 2012. Servei Meteorològic de Catalunya. Departament de Territori i Sostenibilitat. Generalitat de Catalunya.

Esteban P., Prohom Duran M. \& Aguilar E. 2012. Tendencias recientes e índices de cambio climático de la temperatura y la precipitación en Andorra, Pirineos (1935-2008). Pirineos,
167: 89-108. doi: http://dx.doi.org/10.3989/Pirineos.2012. 167005

Fukushima, T., Kureha, M., Ozaki, N., Fujimori, Y. \& Harasawa, H. 2003. Influences of air temperature change on leisure industries: case study on ski activities. Mitigation and Strategies for Climate Change, 7: 173-189.

Gössling, S., D. Scott, C., Michael Hall, J.P., Ceron \& Dubois, G. 2012. Consumer behaviour and demand response of tourists to climate change. Annals of Tourism Research, 39(1): 36-58. http://dx.doi.org/10.1016/j.annals.2011.11.002

Hennessy, K., Whetton, P., Smith, I., Bathols, J., Hutchinson, M. \& Sharples, J. 2003. The impact of climate change on snow conditions in mainland Australia. CSIRO Atmospheric Research., 47 pp., Aspendale, Australia.

Hendrikx, J., Hreinsson, E. Ö, Clark, M.P. \& Mullan, A.B. 2012. The potential impact of climate change on seasonal snow in New Zealand: part I-an analysis using 12 GCMs. Theoretical and Applied Climatology, 110 (4): 607-618. http:// dx.doi.org/10.1007/s00704-012-0711-1

Intergovernmental Panel on Climate Change IPCC 2007. Climate change 2007: synthesis report. Summary for policy makers. Fourth Assessment Report. United Nations Intergovernmental Panel on Climate Change, Geneva.

Intergovernmental Panel on Climate Change IPCC 2013. Climate change 2013: synthesis report. Summary for policy makers. Fifht Assessment Report. United Nations Intergovernmental Panel on Climate Change, Geneva

Keller, F., Kienast, F. \& Beniston, M. 2000. Evidence of the Response of Vegetation to Environmental Change at High Elevation Sites in the Swiss Alps. Regional Environmental Change, 2: 70-77. http://dx.doi.org/10.1007/PL00011535.

Keller, F., Goyette, S. \& Beniston, M. 2005. Sensitivity analysis of snow cover to climate change scenarios and their impact on plant habitats in alpine terrain. Climatic Change, 72 : 299-319. doi: http://dx.doi.org/10.1007/PL00011535

König, U. \& Abegg, B. 1997. Impacts of climate change on tourism in the Swiss Alps. Journal of Sustainable Tourism, 5(1): $46-58$ http://dx.doi.org/10.1080/09669589708667275

Lamothe and Périard Consultants 1988. Implications of climate change for downhill skiing in Québec. Climate Change Digest 88-03. Environment Canada, Ottawa

Lasanta, T., Laguna Marín-Yaseli, M. \& Vicente-Serrano, S.M. 2007. Do tourism-based ski resorts contribute to the homogeneous development of the Mediterranean mountains? A case study in the Central Spanish Pyrenees. Tourism Management, 28(5): 1326-1339. http://dx.doi.org/10.1016/j. tourman.2007.01.003

Lasanta, T., Laguna Marín-Yaseli, M. \& Vicente-Serrano, S.M. 2007b. Variabilidad espacial de los efectos socioeconómicos de las explotaciones de esquí alpino en los municipios rurales del Pirineo aragonés. Pirineos, 162: 155-176.

López-Moreno JI, Goyette S. \& Beniston M 2009. Impact of climate change on snowpack in the Pyrenees: horizontal spatial variability and vertical gradients. Journal Hydrology, 374: 384-396. http://dx.doi.org/10.1016/j.jhydrol.2009. 06.049

McBoyle, G. \& Wall, G. 1987. The impact of CO2 induced warming on downhill skiing in the Laurentians. Cahiers de Géographie du Québec, 31(82): 39-50. http://dx.doi. org/10.7202/021843ar

Moen, J. \& Fredman, P. 2007. Effects of Climate Change on Alpine Skiing in Sweden. Journal of Sustainable Tourism 15(4): 418-437. http://dx.doi.org/10.2167/jost624.0

Pons-Pons, M., Johnson, P.A., Rosas-Casals, M., Sureda, B. \& Jover, È. 2012. Modeling climate change effects on winter ski tourism in Andorra. Climate Research, 54:197-207. http://dx.doi.org/10.3354/cr01117. 
SCAMPEI 2012: Scénarios climatiques adaptés aux zones de motagnes: Phénomènes extrêmes, enneigement et in Certitudes. http://www.cnrm.meteo.fr/scampei/

Scott, D., McBoyle, G. \& Mills, B. 2003. Climate change and the skiing industry in southern Ontario (Canada): Exploring the importance of snowmaking as a technical adaptation. Climate Research, 23: 171-181. http://dx.doi.org/10.3354/ cr023171

Scott, D., McBoyle, G. \& Minogue, A. 2007. Climate change and Quebec's ski industry. Global Environmental Change, 17: 181-190. http://dx.doi.org/10.1016/j.gloenvcha.2006. 05.004

Scott, D., Gössling, S. \& Hall, C.M. 2012. Climate Change and Tourism: Impacts, Adaptation and Mitigation. London: Routledge. 423 pgs.

Ski Andorra. www.skiandorra.ad. Last access November 21st 2013

Steiger R. \& Mayer M. 2008. Snowmaking and climate change. Future options for snow production in Tyrolean ski resorts. Mountain Research and Development, 28: 292-298. http:// dx.doi.org $/ 10.1659 / \mathrm{mrd} .0978$

Steiger, R. 2010. The impact of climate change on ski season length and snowmaking requirements in Tyrol, Austria. Climate Research, 43(3): 251-262. http://dx.doi.org/10.3354/cr00941
Steiger, R. 2012. Scenarios for skiing tourism in Austria: integrating demographics with an analysis of climate change. Journal of Sustainable Tourism, 20(6): 867-882. http:// dx.doi.org/10.1080/09669582.2012.680464

Steiger, R. \& Abegg. B. 2013. The sensitivity of Austrian Ski areas to climate change. Tourism, Planning and Development, 10(4): 480-493. http://dx.doi.org/10.1080/21568316.2 013.804431

Uhlmann, B., Goyette, S. \& Beniston, M. 2009. Sensitivity analysis of snow patterns in Swiss ski resorts to shifts in temperature, precipitation and humidity under condition of climate change. International Journal of Climatology, 29: 1048-1055. http://dx.doi.org/10.1002/joc.1786

Vanat, L. 2013. 2013 International Report On Snow \& Mountain Tourism.

Witmer, U. 1986. Erfassung, Bearbeitung und Kartierung von Schneedaten in der Schweiz. GeographicaBernensia G25.

WTO-UNEP 2003. Climate change and tourism. Proceedings of the First International Conference on Climate change and Tourism, Djerba, 9-11 April. Madrid: World Tourism Organization. 